\title{
A Comparison of a Priori Estimates of the Solutions of a Linear Fractional System with Distributed Delays and Application to the Stability Analysis
}

\author{
Hristo Kiskinov*(D), Magdalena Veselinova, Ekaterina Madamlieva (D) and Andrey Zahariev (D) \\ Faculty of Mathematics and Informatics, University of Plovdiv, 4000 Plovdiv, Bulgaria; \\ veselinova@uni-plovdiv.bg (M.V.); ekaterinaa.b.m@gmail.com (E.M.); zandrey@uni-plovdiv.bg (A.Z.) \\ * Correspondence: kiskinov@uni-plovdiv.bg
}

check for

updates

Citation: Kiskinov, H.; Veselinova, M.; Madamlieva, E.; Zahariev, A. A Comparison of a Priori Estimates of the Solutions of a Linear Fractional System with Distributed Delays and Application to the Stability Analysis. Axioms 2021, 10, 75. https://doi.org/ 10.3390 /axioms 10020075

Academic Editor: Jorge E. Macías Díaz

Received: 31 March 2021

Accepted: 21 April 2021

Published: 27 April 2021

Publisher's Note: MDPI stays neutral with regard to jurisdictional claims in published maps and institutional affiliations.

Copyright: (c) 2021 by the authors. Licensee MDPI, Basel, Switzerland. This article is an open access article distributed under the terms and conditions of the Creative Commons Attribution (CC BY) license (https:/ / creativecommons.org/licenses/by/ $4.0 /)$.

\begin{abstract}
In this article, we consider a retarded linear fractional differential system with distributed delays and Caputo type derivatives of incommensurate orders. For this system, several a priori estimates for the solutions, applying the two traditional approaches-by the use of the Gronwall's inequality and by the use of integral representations of the solutions are obtained. As application of the obtained estimates, different sufficient conditions which guaranty finite-time stability of the solutions are established. A comparison of the obtained different conditions in respect to the used estimates and norms is made.
\end{abstract}

Keywords: Caputo fractional derivative; linear fractional system; distributed delay; finite time stability

MSC: 34A08; 34A30; 26A33; 34A12

\section{Introduction.}

As a highly applicable mathematical tool to study models of real-world phenomena, fractional calculus theory attracts a lot of attention. For a deep understanding of the fractional calculus theory and fractional differential equations, we recommend the monographs [1,2]. The distributed order fractional differential equations are treated in [3], and for an application-oriented exposition see [4]. The impulsive functional differential equations and some applications are considered in [5]. Some new ideas for efficient schemes for numerical solving of fractional differential problems can be found, for example, in [6,7].

Fractional differential equations with delay generally speaking are more complicated in comparison with the integer order differential equations with delay. This is conditioned such that a distinguishing feature of the fractional differential equations with delay is that the evolution of the processes described by such equations depends on the past history inspired from two independent sources. The first of them is the impact condition of the delays and the other one the impact condition from the availability of Volterra type integral in the definitions of the fractional derivatives, i.e., the memory of the fractional derivative.

It is well known that the classical stability concepts (Lyapunov type stabilities) are devoted to study the asymptotical properties of the solutions of differential systems over an infinite time interval. It is well known that the theme of the stability of the solutions of fractional differential equations and/or systems (ordinary or with delay) is an "evergreen" theme for research. Furthermore, the wide appearance of the aftereffect to regard it as a universal property of the surrounding world, is a serious reason to consider mathematical models with delay and fractional derivatives. This explains why a lot of papers are devoted to different aspects of this problem. A very good overview of the stability of the fractional differential systems is given in the comprehensive survey [8]. From the recent works we refer also to [9-18].

However, in many practical cases is more important to study the solution behaviors in some specified (finite) time interval, where larger values of the state variables are not 
admissible. Moreover, many authors made the observation that a system could be stable, but it can own unacceptable transient outputs. Such a situation from an engineering point of view leads to these types of analysis being useless. This is a reason to study not only Lyapunov type stabilities but also to study the boundedness of the solutions defined over a finite time interval, i.e., the finite-time stability (FTS). As far as we know the first work concerning the FTS is written by Kamenkov [19] in the year 1953. A historical overview of this theme can be obtained from the survey of Dorato [20]. Concerning the more recent works devoted to the different approaches to study the finite-time stability, we refer to the works [21-30].

The aim of our work, motivated by remarkable works [24-27], is twofold. First, we obtain a priori estimates using the two most popular approaches and then compare the precisions of the obtained via them estimates. Second, as an application, we apply these estimates to investigate the finite-time stability of fractional differential systems with Caputo type derivatives in the case of incommensurate fractional orders and distributed delays.

The paper is organized as follows. In Section 2, we recall the definitions of Riemann-Liouville and Caputo fractional derivatives. In the same section is the statement of the problem, as well as some necessary definitions and preliminary results used later. Section 3 is devoted to obtaining a priori estimates of the solutions of nonautonomous fractional differential systems with Caputo type derivatives of incommensurate orders with distributed delays via Gronwall inequality. In Section 4 for the solutions of the same systems we obtain a priori estimates using the approach based on their integral representations obtained in [31]. In Section 5 as application of the proved estimates we obtain sufficient conditions for finite-time stability of the considered systems. Some examples and comments are given in Section 5 and in Section 6 we present conclusions about the two main approaches analyzed in the previous sections.

\section{Preliminaries and Problem Statement}

For the reader convenience, below we recall the definitions of Riemann-Liouville and Caputo fractional derivatives. For details and properties we refer to [1-3].

Let $\alpha \in(0,1)$ be an arbitrary number and denote by $L_{1}^{\text {loc }}(\mathbb{R}, \mathbb{R})$ the linear space of all locally Lebesgue integrable functions $f: \mathbb{R} \rightarrow \mathbb{R}$. Then for $a \in \mathbb{R}, f \in L_{1}^{\text {loc }}(\mathbb{R}, \mathbb{R})$ and each $t>a$ the definitions of the left-sided fractional integral operator, the left side Riemann-Liouville and Caputo fractional derivatives of order $\alpha$ with lower limit (terminal) $a$ are given below (see [1]):

$$
\begin{gathered}
\left(D_{a+}^{-\alpha} f\right)(t)=\frac{1}{\Gamma(\alpha)} \int_{a}^{t}(t-s)^{\alpha-1} f(s) \mathrm{d} s, \\
R L D_{a+}^{\alpha} f(t)=\frac{\mathrm{d}}{\mathrm{d} t}\left(D_{a+}^{-(1-\alpha)} f(t)\right) ; \\
{ }_{C} D_{a+}^{\alpha} f(t)={ }_{R L} D_{a+}^{\alpha}[f(s)-f(a)](t) ;
\end{gathered}
$$

Everywhere below the following notations will be used: $\mathbb{R}_{+}=(0, \infty), \overline{\mathbb{R}}_{+}=[0, \infty)$, $J_{T}=[0, T], T \in \mathbb{R}_{+},\langle n\rangle=\{1,2, \ldots, n\},\langle n\rangle_{0}=\langle n\rangle \cup\{0\}, n \in \mathbb{N}, I, \Theta \in \mathbb{R}^{n \times n}$ denote the identity and zero matrix respectively, $I^{k}, k \in\langle n\rangle$ denotes the $k$-th column of the identity matrix and $\mathbf{0} \in \mathbb{R}^{n}$ is the zero element.

For $\beta=\left(\beta_{1}, \ldots, \beta_{n}\right), \beta_{k} \in[-1,1], k \in\langle n\rangle, Y(t)=\left(y^{1}(t), \ldots, y^{n}(t)\right)^{T}: \mathbb{R}_{+} \rightarrow \mathbb{R}^{n}$ we use the notations $I_{\beta}(Y(t))=\operatorname{diag}\left(\left(y_{1}(t)\right)^{\beta_{1}}, \ldots,\left(y_{n}(t)\right)^{\beta_{n}}\right)$, for $W(t)=\left\{w_{k j}(t)\right\}_{k, j=1}^{n}$ : $\overline{\mathbb{R}}_{+} \rightarrow \mathbb{R}^{n \times n}, W(t) \in L_{1}^{\text {loc }}\left(\overline{\mathbb{R}}_{+}, \mathbb{R}^{n \times n}\right)$ and is locally bounded, we note for every fixed $t \in \overline{\mathbb{R}}_{+}$with $W^{T}(t)=\left\{w_{j k}(t)\right\}_{k, j=1}^{n}$ the transposed matrix, with $\sigma^{\operatorname{Max}}(t)$ the largest singular value of $W(t)$ and with $|W(t)|=\sigma^{M a x}$ the spectral norm [32]. In addition, $\|W(t)\|=$ sup $|W(\xi)|, t \in \overline{\mathbb{R}}_{+}$and for simplicity we will use the notation $D_{0+}^{\alpha}={ }_{C} D_{0+}^{\alpha}$ for the left $\xi \in[0, t]$

side Caputo fractional derivative with lower terminal zero. 
Below we will study the inhomogeneous linear delayed system of incommensurate type and distributed delay in the following general form

$$
D_{0+}^{\alpha} X(t)=\int_{-h}^{0}\left[\mathrm{~d}_{\theta} U(t, \theta)\right] X(t+\theta)+F(t), t \in \mathbb{R}_{+}
$$

or described in rows

$$
\left.D_{0+}^{\alpha_{k}} x_{k}(t)=\sum_{j=1}^{n} \int_{-h}^{0} x_{j}(t+\theta) d_{\theta} u_{k j}(t, \theta)\right)+f_{k}(t), t \in \mathbb{R}_{+}, k \in\langle n\rangle
$$

where $X(t)=\left(x_{1}(t), \ldots, x_{n}(t)\right)^{T}, D_{0+}^{\alpha}=\operatorname{diag}\left(D_{0+}^{\alpha_{1}}, \ldots, D_{0+}^{\alpha_{n}}\right), h \in \mathbb{R}_{+}$is an arbitrary fixed number, $\alpha=\left(\alpha_{1}, \ldots, \alpha_{n}\right), \alpha_{k} \in(0,1), U: \overline{\mathbb{R}}_{+} \times \mathbb{R} \rightarrow \mathbb{R}^{n \times n}, \mathcal{U}(t, \theta)=\left\{u_{k j}(t, \theta)\right\}_{k, j=1}^{n}, F(t)=$ $\left(f_{1}(t), \ldots, f_{n}(t)\right)^{T}: \bar{R}_{+} \rightarrow \mathbb{R}^{n}, \alpha_{M}=\max _{k \in\langle n\rangle} \alpha_{k}$ and $\alpha_{m}=\min _{k \in\langle n\rangle} \alpha_{k}$.

Definition 1. With $\tilde{C}$ we denote the Banach space of all bounded vector functions $\Phi(t) \in$ $L_{1}^{\text {loc }}\left([-h, 0], \mathbb{R}^{n}\right)$, with finite many jumps and norm $\|\Phi\|=\sup _{t \in[-h, 0]}|\Phi(t)|=\max _{k \in\langle n\rangle}\left(\sup _{t \in[-h, 0]}\left|\phi_{k}(t)\right|\right)$ $<\infty$ and the subspace of all continuous functions by $C=C\left([-h, 0], \mathbb{R}^{n}\right)$, i.e., $C \subset \tilde{\boldsymbol{C}}$. Below we assume for convenience, that every $\Phi \in \tilde{C}$ is prolonged as $\Phi(t)=\mathbf{0}$ for $t \in(-\infty,-h)$ and by $S^{\Phi}$ we will denote the set of the jump points of $\Phi$.

For the system, (1) introduces the following initial conditions:

$$
X(t)=\Phi(t)\left(x_{k}(t)=\phi_{k}(t), k \in\langle n\rangle\right), \quad t \in(-\infty, 0], \Phi \in \tilde{C} .
$$

We say that for the kernel $U: \overline{\mathbb{R}}_{+} \times \mathbb{R} \rightarrow \mathbb{R}^{n \times n}$ the conditions (S) hold for some $h \in \mathbb{R}_{+}$if the following conditions are fulfilled:

(S1) The functions $(t, \theta) \rightarrow U(t, \theta)=\left\{u_{k j}(t, \theta)\right\}_{k, j=1}^{n}$ are measurable in $(t, \theta) \in \overline{\mathbb{R}}_{+} \times \mathbb{R}$ and normalized so that for $t \in \overline{\mathbb{R}}_{+}, U(t, \theta)=0$ when $\theta \in \overline{\mathbb{R}}_{+}$and $U(t, \theta)=U(t,-h)$ for all $\theta \in(-\infty,-h]$. For all $t \in \overline{\mathbb{R}}_{+}$the matrix valued function $\bar{U}(t, 0)=$ $\operatorname{Var}_{\theta \in[-h, 0]} U(t, \theta)=\left\{\operatorname{Var}_{\theta \in[-h, 0]} u_{k, j}(t, \theta)\right\}_{k, j=1}^{n}, \bar{U}(t, 0) \in L_{1}^{\text {loc }}\left(\mathbb{R}_{+}, \mathbb{R}^{n \times n}\right)$ is locally bounded and $\max _{k, j \in\langle n\rangle} \operatorname{Var}_{\theta \in[-h, 0]} u_{k, j}(t, \theta)<\infty$.

(S2) The Lebesgue decomposition of the kernel $U(t, \theta)$ for $t \in \overline{\mathbb{R}}_{+}$and $\theta \in[-h, 0]$ has the form:

$$
U(t, \theta)=U_{J}(t, \theta)+U_{A C}(t, \theta)+U_{S}(t, \theta)
$$

where $U_{J}(t, \theta)=\sum_{i=0}^{m} A^{i}(t) H\left(\theta+\sigma_{i}(t)\right), m \in \mathbb{N}, A^{i}(t)=\left\{a_{k j}^{i}(t)\right\}_{k, j=1}^{n} \in L_{1}^{l o c}\left(\mathbb{R}_{+}, \mathbb{R}^{n \times n}\right)$ are locally bounded on $\mathbb{R}_{+}, H(t)$ is the Heaviside function, the delays $\sigma_{i}(t) \in C\left(\overline{\mathbb{R}}_{+}, \overline{\mathbb{R}}_{+}\right)$are bounded with $\sigma_{i}=\sup _{t \in \overline{\mathbb{R}}_{+}} \sigma_{i}(t), \max _{i \in\langle m\rangle} \sigma_{i}=h, i \in\langle m\rangle, \sigma_{0}(t) \equiv 0, U_{A C}=\left\{\int_{-h}^{\theta} b_{k}^{j}(t, s) \mathrm{d} s\right\}_{k, j=1}^{n} \in$ $L_{1}^{\text {loc }}\left(\overline{\mathbb{R}}_{+} \times \mathbb{R}, \mathbb{R}^{n \times n}\right)$ are locally bounded on $\overline{\mathbb{R}}_{+}$and $U_{S}(t, \theta) \in C\left(\overline{\mathbb{R}}_{+} \times \mathbb{R}, \mathbb{R}^{n \times n}\right)$.

(S3) For every $t^{*} \in \mathbb{R}_{+}$the following relation hold: $\lim _{t \rightarrow t_{*}} \int_{-h}^{0}\left|U(t, \theta)-U\left(t_{*}, \theta\right)\right| \mathrm{d} \theta=0$.

(S4) The set $S_{U}=\left\{t \in \overline{\mathbb{R}}_{+} \mid t-\sigma_{i}(t) \in S^{\Phi}, i \in\langle m\rangle\right\}$ do not have limit points.

Remark 1. At first glance, it seems that condition (S4) imposes certain restrictions on the initial function (more preciously on its jump set $S^{\Phi}$, which is a finite set). But the leading role in this interaction belongs to the delays, i.e., the validity of (S4) depends only from the properties of the delays. For example, in the cases of constant delays or when the delays are strictly increasing, then (S4) is ultimately fulfilled. 
Let us consider the following auxiliary system in matrix form

$$
\begin{array}{r}
X(t)=\Phi(0)+I_{-1}(\Gamma(\alpha))\left[\int_{0}^{t} I_{\alpha-1}(t-\eta) \int_{-h}^{0}\left[\mathrm{~d}_{\theta} U(\eta, \theta)\right] X(\eta+\theta) \mathrm{d} \eta+\int_{0}^{t} I_{\alpha-1}(t-\eta) F(\eta) \mathrm{d} \eta\right] \\
\text { where } I_{-1}(\Gamma(\alpha))=\operatorname{diag}\left(\Gamma^{-1}\left(\alpha_{1}\right), \ldots, \Gamma^{-1}\left(\alpha_{n}\right)\right), \text { or for } k \in\langle n\rangle \text { in row form } \\
x_{k}(t)=\phi_{k}(0)+\frac{1}{\Gamma\left(\alpha_{k}\right)}\left[\int_{0}^{t}(t-\eta)^{\alpha_{k}-1}\left(\sum_{j=1}^{n} \int_{-h}^{0} x_{j}(\eta+\theta) \mathrm{d}_{\theta} u_{k j}(\eta, \theta)\right) \mathrm{d} \eta+\int_{0}^{\alpha_{k}-1}\left(t-\eta f_{k}(\eta) \mathrm{d} \eta\right]\right.
\end{array}
$$

with the initial condition (2).

In our exposition below we will use the abbreviation IP for Initial Problem.

Definition 2. The vector function $X(t)=\left(x_{1}(t), \ldots, x_{n}(t)\right)^{T}$ is a solution of the IP (1), (2) or IP (3), (2) in $\overline{\mathbb{R}}_{+}$, if $X \in C\left(\overline{\mathbb{R}}_{+}, \mathbb{R}^{n}\right)$ satisfies the system (1) respectively (3) for all $t \in \mathbb{R}_{+}$and the initial condition (2) for each $t \in[-h, 0]$.

In virtue of Lemma 3.3 in [33] every solution $X(t)$ of IP (1), (2) is a solution of IP (3), (2) and vice versa. Moreover, the IP (3), (2) possess a unique solution $X \in C\left(\overline{\mathbb{R}}_{+}, \mathbb{R}^{n}\right)$ according Corollary 1 in [34] and hence IP (1), (2) too.

For the corresponding homogeneous system of the system (1) (i.e., $F(t) \equiv \mathbf{0}$ for $\left.t \in \mathbb{R}_{+}\right)$:

$$
D_{0+}^{\alpha} X(t)=\int_{-h}^{0}\left[\mathrm{~d}_{\theta} U(t, \theta)\right] X(t+\theta), t \in \mathbb{R}_{+}
$$

and for arbitrary fixed $s \in[-h, \infty)$ introduce the matrix system

$$
D_{0+}^{\alpha} W(t, s)=\int_{-h}^{0}\left[\mathrm{~d}_{\theta} U(t, \theta)\right] W(t+\theta, s), t \in \mathbb{R}_{+} \cap[s, \infty) .
$$

as well as the special kind initial matrix valued functions $\Phi_{1}, \Phi_{2}: \mathbb{R}^{2} \rightarrow \mathbb{R}^{n \times n}$

$$
\begin{aligned}
& \Phi_{1}(t, s)=\left\{\begin{array}{ll}
I, & t=s, \\
\Theta, & t<s
\end{array}, s \in \overline{\mathbb{R}}_{+,}\right. \\
& \Phi_{2}(t, s)=\left\{\begin{array}{ll}
I, & -h \leq s \leq t \leq 0, \\
\Theta, & t<s \text { or } s<-h
\end{array}, s \in[-h, 0]\right.
\end{aligned}
$$

and consider the matrix integral equations

$$
\begin{aligned}
C(t, s) & =\Phi_{1}(t, s)+I_{-1}(\Gamma(\alpha)) \int_{s}^{t} I_{\alpha-1}(t-\eta) \int_{-\sigma}^{0}[\mathrm{~d} U(\eta, \theta)] C(\eta+\theta, s) \mathrm{d} \eta, s \in \overline{\mathbb{R}}_{+}, t \in(s, \infty) \\
T_{-h}(t, s) & =\Phi_{2}(0, s)+I_{-1}(\Gamma(\alpha)) \int_{0}^{t} I_{\alpha-1}(t-\eta) \int_{-\sigma}^{0}[\mathrm{~d} U(\eta, \theta)] T_{-h}(\eta+\theta, s) \mathrm{d} \eta, s \in[-h, 0], t \in \mathbb{R}_{+}
\end{aligned}
$$

For arbitrary fixed $s \in \overline{\mathbb{R}}_{+}$, the solution $C(t, s)$ of $(7)$ for $t \in(s, \infty)$ with initial condition $C(t, s)=\Phi_{1}(t, s), t \in(-\infty, s]$ is called fundamental matrix of the system (4).

By $T_{-h}(t, s)$ for arbitrary fixed $s \in(-\infty, 0]$ we denote the solution of (8) for $t \in \mathbb{R}_{+}$ with initial condition $T_{-h}(t, s)=\Phi_{2}(t, s), t \in(-\infty, 0]$ and we note that $C(t, 0)=T_{0}(t, 0)$.

The existence and uniqueness of the fundamental matrix $C(t, s)$ of the system (4) and the matrix $T_{-h}(t, s)$ as well as their properties are proved in [31]. Please note that these matrices are absolutely continuous concerning $t$ and continuous in $s$ on every compact subinterval in $\overline{\mathbb{R}}_{+}$if $s \neq t$ and for $s=t$ possess first kind jumps [31]. 
Everywhere below we will use the notations:

$$
\begin{gathered}
\|\bar{U}(t, 0)\|=\sup _{\xi \in[0, t]}|\bar{U}(\xi, 0)|=\sup _{\xi \in[0, t]}\left|\operatorname{Var}_{\theta \in[-h, 0]} U(\xi, \theta)\right|, \\
\bar{C}(t, s)=\operatorname{Var}_{\eta \in[0, s]} C(t, \eta)=\left\{\operatorname{Var}_{\eta \in[0, s]} c_{k j}(t, \eta)\right\}_{k, j=1}^{n}, \\
\|\bar{C}(t, s)\|=\sup _{\xi \in[0, t]}|\bar{C}(\xi, s)|=\sup _{\xi \in[0, t]}\left|\operatorname{Var}_{\eta \in[0, s]} C(\xi, \eta)\right|, \\
\bar{T}_{-h}(t, s)=\operatorname{Var}_{\eta \in[-h, s]} T_{-h}(t, \eta)=\left\{\operatorname{Var}_{\eta \in[-h, s]} \vartheta_{k j}(t, \eta)\right\}_{k, j=1}^{n}, \\
\left\|\bar{T}_{-h}(t, s)\right\|=\sup _{\xi \in[0, t]}\left|\bar{T}_{-h}(\xi, s)\right|=\sup _{\xi \in[0, t]}\left|\operatorname{Var}_{\eta \in[-h, s]} \bar{T}_{-h}(\xi, \eta)\right| .
\end{gathered}
$$

We recall some needed properties of the gamma function $\Gamma(z), z \in \mathbb{R}_{+}$.

It is well known that $\Gamma(z)$ has a local minimum at $z_{\min } \approx 1.46163$, where it attains the value $\Gamma\left(z_{\min }\right) \approx 0.885603$. Since $\Gamma(z)$ for $z \in\left(0, z_{\min }\right)$ is strictly decreasing, then for arbitrary $\alpha_{k} \in(0,1)$ we have that

$$
\max _{k \in\langle n\rangle} \frac{1}{\Gamma\left(\alpha_{k}\right)}<\max _{k \in\langle n\rangle} \frac{1}{\Gamma\left(1+\alpha_{k}\right)} \leq \frac{1}{\Gamma\left(z_{\min }\right)} \leq 1.1279
$$

For the function $I_{\alpha-1}(t-\eta)=\left(\operatorname{diag}\left((t-\eta)^{\alpha_{1}-1}, \ldots,(t-\eta)^{\alpha_{n}-1}\right)\right.$ we will use below the notations $\alpha_{*}=\alpha_{m}$ when $t-\eta \leq 1$ and $\alpha_{*}=\alpha_{M}$ when $t-\eta \geq 1$. Then we have that for $t \in \overline{\mathbb{R}}_{+}, \eta \in[0, t)$, the following relations hold

$$
\left|I_{\alpha-1}(t-\eta)\right|=(t-\eta)^{\alpha_{*}-1} ;\left|I_{-1}(\Gamma(\alpha))\right|=\frac{1}{\Gamma\left(\alpha_{M}\right)}=\Gamma^{-1}\left(\alpha_{M}\right)=C_{0}
$$

where $\Gamma^{-1}\left(\alpha_{M}\right)$ and $(t-\eta)^{\alpha_{*}-1}$ are the largest singular values for the diagonal matrices $I_{-1}(\Gamma(\alpha))$ and $I_{\alpha-1}(t-\eta)$ respectively.

Theorem 1. [35] Let the following conditions hold:

1. The functions $a(t), u(t) \in L_{\text {loc }}^{1}\left([0, T), \overline{\mathbb{R}}_{+}\right)$for some $T \in \mathbb{R}_{+}$and $\alpha>0$.

2. The function $g(t) \in C([0, T),[0, M])$ for some $M \in \mathbb{R}_{+}$and is nondecreasing.

3. For every $t \in[0, T)$ the following inequality holds:

$$
u(t) \leq a(t)+g(t) \int_{0}^{t}(t-\eta)^{\alpha-1} u(\eta) \mathrm{d} \eta .
$$

Then the following inequality holds for $t \in[0, T)$ :

$$
u(t) \leq a(t)+\int_{0}^{t}\left[\sum_{q=1}^{\infty} \frac{\left(g(\eta)(\Gamma(\alpha))^{q}\right.}{\Gamma(\alpha q)}(t-\eta)^{\alpha q-1}\right] a(\eta) \mathrm{d} \eta .
$$

Corollary 1. [35] Let the conditions of Theorem 1 hold and let the function a $(t)$ be nondecreasing on $[0, T)$.

Then for $t \in[0, T)$ the inequality $u(t) \leq a(t) E_{\alpha}\left[g(t) \Gamma(\alpha) t^{\alpha}\right]$ holds, where $E_{\alpha}$ denotes the one parameter Mittag-Leffler function.

Definition 3. [27] The fractional system given by (1) satisfying the initial state (2) is finite-time stable with respect to $\left\{0, J_{T}, \delta, \varepsilon, h\right\}$ with $t \in J_{T}$ and $\delta \leq \varepsilon$ if and only if the inequality $\|\Phi\|<\delta$ implies that $\|X(t)\|<\varepsilon$ for each $t \in J_{T}$, where $X(t)$ is the unique solution of IP (1), (2). 


\section{A Priory Estimates of the Solutions of IP (1), (2)—Gronwall's Inequality Approach}

In this section, we obtain some a priori estimates of the solutions of IP (1), (2) and IP (4), (2) in different cases, depending from the properties of the initial function $\Phi$ and the function $F$. The different a priori estimates of the solutions in this section are obtained using approaches based on Gronwall's inequality.

Theorem 2. Let $T \in \mathbb{R}_{+}$be an arbitrary fixed number and the following conditions are fulfilled:

1. Conditions (S) hold.

2. The function $F(t) \in L_{1}^{\text {loc }}\left(\mathbb{R}_{+}, \mathbb{R}^{n}\right)$ is locally bounded.

Then for every initial function $\Phi \in \tilde{C}$ the corresponding unique solution X( $t$ ) of IP (1), (2) for every $t \in J_{T}$ satisfies the estimation

$$
\max (\|X(t)\|,\|\Phi\|) \leq\left(\|\Phi\|+\alpha_{*}^{-1} C_{0}\|F(t)\| t^{\alpha_{*}}\right) E_{\alpha}\left(\|\bar{U}(t, 0)\| C_{0} \Gamma\left(\alpha_{*}\right) t^{\alpha_{*}}\right) .
$$

Proof. Let $\Phi \in \tilde{C}$ be an arbitrary initial function and $X(t)$ be the corresponding unique solution of the IP (1), (2). Then if $\max (\|X(T)\|,\|\Phi\|)=\|\Phi\|$ the estimation (10) obviously holds.

Let assume that $\max (\|X(T)\|,\|\Phi\|)>\|\Phi\|$. From (3) for every $t \in J_{T}$ it follows that

$$
X(t)=\Phi(0)+I_{-1}(\Gamma(\alpha))\left[\int_{0}^{t} I_{\alpha-1}(t-\eta) F(\eta) \mathrm{d} \eta+\int_{0}^{t} I_{\alpha-1}(t-\eta) \int_{-h}^{0}\left[\mathrm{~d}_{\theta} U(\eta, \theta)\right] X(\eta+\theta) \mathrm{d} \eta\right] .
$$

Using (9) it is simple to check that

$$
\begin{aligned}
\mid I_{-1}(\Gamma(\alpha))\left[\int_{0}^{t} I_{\alpha-1}(t-\eta) F(\eta) \mathrm{d} \eta \mid\right. & \leq \frac{1}{\Gamma\left(\alpha_{M}\right)} \int_{0}^{t}(t-\eta)^{\alpha_{*}-1}|F(\eta)| \mathrm{d} \eta \\
& \leq C_{0}\|F(t)\| \int_{0}^{t}(t-\eta)^{\alpha_{*}-1} \mathrm{~d} \eta=C_{0} \alpha_{*}^{-1}\|F(t)\| t^{\alpha_{*}} .
\end{aligned}
$$

Since for each $\eta \in \overline{\mathbb{R}}_{+}$with $\eta+\theta \leq 0$ for some $\theta \in[-h, 0]$ we have that $\mid X(\eta+$ $\theta) \mid \leq\|\Phi\|$ and for each $\eta \in \overline{\mathbb{R}}_{+}$with $\eta+\theta \in[0, \eta]$ for some $\theta \in[-h, 0]$ the estimation $|X(\eta+\theta)| \leq\|X(\eta)\|$ holds, then for $t \in J_{T}$ we obtain

$$
\left|\int_{-h}^{0}\left[\mathrm{~d}_{\theta} U(\eta, \theta)\right] X(\eta+\theta)\right| \leq\|\bar{U}(t, 0)\| \max (\|X(\eta)\|,\|\Phi\|) .
$$

Then from (9), (11)-(13) for $t \in J_{T}$ we obtain

$$
\begin{aligned}
\max (\|X(t)\|,\|\Phi\|) & \leq\|\Phi\|+C_{0} \int_{0}^{t}(t-\eta)^{\alpha_{*}-1}\|F(t)\| \mathrm{d} \eta \\
& +\frac{1}{\Gamma\left(\alpha_{M}\right)} \int_{0}^{t}(t-\eta)^{\alpha_{*}-1}\left|\int_{-h}^{0}\left[\mathrm{~d}_{\theta} U(\eta, \theta)\right] X(\eta+\theta)\right| \mathrm{d} \eta \\
& \leq\|\Phi\|+C_{0} \alpha_{*}^{-1} t^{\alpha_{*}}\|F(t)\| \\
& \left.+C_{0}\|\bar{U}(t, 0)\| \int_{0}^{t}(t-\eta)^{\alpha_{*}-1} \max (\|X(\eta)\|) \mid,\|\Phi\|\right) \mathrm{d} \eta
\end{aligned}
$$


and denoting $u(t)=\max (\|X(t)\|,\|\Phi\|)$, from (14) it follows that

$$
u(t) \leq\left(\|\Phi\|+C_{0} \alpha_{*}^{-1} t^{\alpha_{*}}\|F(t)\|\right)+C_{0}\|\bar{U}(t, 0)\| \sup _{\xi \in[0, t]} \int_{0}^{\xi}(\xi-\eta)^{\alpha_{*}-1} u(\eta) \mathrm{d} \eta
$$

Since $u(t)$ is positive and non-decreasing then for each $t \in J_{T}$ we have

$$
\begin{aligned}
\sup _{\xi \in[0, t]} \int_{0}^{\xi}(\xi-\eta)^{\alpha_{*}-1} u(\eta) \mathrm{d} \eta & =\sup _{\xi \in[0, t]} \int_{0}^{\xi} s^{\alpha_{*}-1} u(\xi-s) \mathrm{d} s \\
& \leq \int_{0}^{t} s^{\alpha_{*}-1} u(t-s) \mathrm{d} s=\int_{0}^{t}(t-\eta)^{\alpha_{*}-1} u(\eta) \mathrm{d} s
\end{aligned}
$$

and hence from (15) and (16) it follows for each $t \in J_{T}$ the estimation

$$
u(t) \leq\left(\|\Phi\|+C_{0} \alpha_{*}^{-1} t^{\alpha_{*}}\|F(t)\|\right)+C_{0}\|\bar{U}(t, 0)\| \int_{0}^{t}(t-\eta)^{\alpha_{*}-1} u(\eta) \mathrm{d} s
$$

Then applying Corollary 1 to (17) we obtain (10).

Corollary 2. Let $T \in \mathbb{R}_{+}$be an arbitrary fixed number and the following conditions are fulfilled:

1. The conditions (S) hold.

2. $\|F(T)\|=0$.

Then for every initial function $\Phi \in \tilde{C}$ with $\|\Phi\|>0$ the corresponding unique solution $X(t)$ of IP (1), (2) for every $t \in J_{T}$ satisfies the estimation

$$
\max (\|X(t)\|,\|\Phi\|) \leq\|\Phi\| E_{\alpha}\left(\|\bar{U}(t, 0)\| C_{0} \Gamma\left(\alpha_{*}\right) t^{\alpha_{*}}\right) .
$$

Proof. The estimation (18) follows immediately from (10) using that $\|F(t)\|=0$ for each $t \in[0, T]$.

Corollary 3. Let $T \in \mathbb{R}_{+}$be an arbitrary fixed number and the following conditions are fulfilled:

1. The conditions $(S)$ hold.

2. The function $F(t) \in L_{1}^{\text {loc }}\left(\mathbb{R}_{+}, \mathbb{R}^{n}\right)$ is locally bounded and $\|\Phi\|=0$.

Then the corresponding unique solution X(t) of IP (1), (2) satisfies the estimation

$$
\|X(t)\| \leq \alpha_{*}^{-1} C_{0} t^{\alpha_{*}}\|F(t)\| E_{\alpha}\left(\|\bar{U}(t, 0)\| C_{0} \Gamma\left(\alpha_{*}\right) t^{\alpha_{*}}\right) .
$$

Proof. The estimation (19) follows immediately from (10) using that $\|\Phi\|=0$.

The next theorem is devoted to obtaining another form of the estimation (10) based on the assumption that $\|\Phi\|>0$. The approach used is the same as in Theorem 2 but the assumption that $\|\Phi\|>0$ allows one technical stunt to be realized.

Theorem 3. Let $T \in \mathbb{R}_{+}$be an arbitrary fixed number and the following conditions are fulfilled:

1. The condition of Theorem 2 hold and $\|F(T)\|>0$.

2. The initial function $\Phi \in \tilde{C}$ satisfies the condition $\|\Phi\|>0$.

Then the corresponding unique solution $X(t)$ of IP (1), (2) for every $t \in J_{T}$ satisfies the estimation

$$
\max (\|X(t)\|,\|\Phi\|) \leq\|\Phi\| E_{\alpha}\left(\left(C_{\Phi}+\|\bar{U}(t, 0)\|\right) C_{0} \Gamma\left(\alpha_{*}\right) t^{\alpha_{*}}\right),
$$

where $C_{\Phi}=\|\Phi\|^{-1}\|F(T)\|$. 
Proof. Let $X(t)$ be the corresponding unique solution of the IP (1), (2). Condition 2 implies that $\|\Phi\|>0$ and then since $\sup _{s \in[-h, t]}|X(s)|$ is not decreasing and $X(t)=\Phi(t)$ for $t \in[-h, 0]$, then we have that $\sup |X(t)|=\|\Phi\|$. Let assume that $\|\Phi\|>|X(0)|$ and let $\bar{t} \in[0, T]$ be arbitrary with $\|\Phi\| \geq|X(\bar{t})|$. Then for $C_{\Phi}=\|\Phi\|^{-1}|| F(T) \|$ we have

$$
C_{\Phi} \max (|X(\bar{t})|,\|\Phi\|)=C_{\Phi}\|\Phi\|=\|\Phi\| \frac{\|F(T)\|}{\|\Phi\|}=\|F(T)\| \geq\|F(\bar{t})\| \geq|F(\bar{t})| .
$$

For arbitrary $\bar{t} \in[0, T]$ with $\|\Phi\| \leq|X(\bar{t})|$ we obtain that the inequality

$$
C_{\Phi} \max (|X(\bar{t})|,\|\Phi\|)=C_{\Phi}|X(\bar{t})| \geq\|\Phi\| \frac{\|F(T)\|}{\|\Phi\|}=\|F(T)\| \geq\|F(\bar{t})\| \geq|F(\bar{t})|
$$

holds and hence for each $t \in J_{T}$ the inequality $\|F(t)\| \leq C_{\Phi} \max (|X(t)|,\|\Phi\|)$ holds.

Then for each $t \in J_{T}$ from (11) as in the proof of Theorem 2 we obtain that (14) holds.

From (14) and taking into account the inequality $\|F(t)\| \leq C_{\Phi} \max (|X(t)|,\|\Phi\|)$ it follows that

$$
\begin{aligned}
\max (|X(t)|,\|\Phi\|) & \leq\|\Phi\|+C_{0} C_{\Phi} \int_{0}^{t}(t-\eta)^{\alpha_{*}-1} \max (|X(\eta)|,\|\Phi\|) \mathrm{d} \eta \\
& +C_{0}\|\bar{U}(t, 0)\| \int_{0}^{t}(t-\eta)^{\alpha_{*}-1} \max (|X(\eta)|,\|\Phi\|) \mathrm{d} \eta \\
& \leq\|\Phi\|+C_{0}\left(C_{\Phi}+\|\bar{U}(t, 0)\|\right) \int_{0}^{t}(t-\eta)^{\alpha_{*}-1} \max (|X(\eta)|,\|\Phi\|) \mathrm{d} \eta
\end{aligned}
$$

and hence from (21) as in the proof of Theorem 2 we obtain

$$
u(t) \leq\|\Phi\| C_{0}\left(C_{\Phi}+\|\bar{U}(t, 0)\|\right) \int_{0}^{t}(t-\eta)^{\alpha_{*}-1} u(\eta) \mathrm{d} \eta .
$$

Then applying Corollary 1 to (22) we obtain (20).

Remark 2. At first glance, it looks like the estimate (20) is better at least as it has a more appropriate form for the applications in compare with (10). However, the most important question is which estimate is more accurate since in general the approach used in both proofs is the same. It is simple to establish that if $\|\Phi\|=0$ then the estimate (19) can be used and in the case when $\|\Phi\|>0$ the estimate (10) can be rewritten in the form

$$
\max (\|X(t)\|,\|\Phi\|) \leq\|\Phi\|\left(1+\alpha_{*}^{-1} C_{0} C_{\Phi} t^{\alpha_{*}}\right) E_{\alpha}\left(\|\bar{U}(t, 0)\| C_{0} \Gamma\left(\alpha_{*}\right) t^{\alpha_{*}}\right) .
$$

These simple considerations limit the impact to linear (no more than power-law) growth in the right side of the estimation (23) and allow avoiding the high nonlinear impact of $C_{\Phi}=$ $\|\Phi\|^{-1}\|F(T)\|$ as argument in the Mittag-Leffler function $E_{\alpha}(\cdot)$ in (20).

\section{A Priory Estimates of the Solutions Obtained via Their Integral Representations}

The next different a priori estimations are obtained using the other most popular approach, which is essentially based on the different kinds integral representations of the solutions of the considered systems obtained in [31,33] and applying the superposition principle. 
Theorem 4. Let $T \in \mathbb{R}_{+}$be an arbitrary fixed number and following conditions are fulfilled:

1. The conditions of Theorem 2 hold.

2. The initial function $\Phi(t) \equiv \mathbf{0}$ for $t \in[-h, 0]$ (i.e., $\|\Phi\|=0$ ).

Then the corresponding unique solution $X^{F}(t)$ of the IP (1), (2) for every $t \in J_{T}$ satisfies the estimation

$$
\left\|X^{F}(t)\right\| \leq \alpha_{*}^{-1} t^{\alpha_{*}} C_{0}\|F(t)\|(1+\|\bar{C}(t, t)\|) .
$$

Proof. Let $\Phi \in \tilde{C}$ and $\Phi(t) \equiv \mathbf{0}$ for $t \in[-h, 0]$. Then according Theorem 4.3 in [33] the unique solution $X^{F}(t)$ of the IP (1), (2) for every $t \in \mathbb{R}_{+}$has the following representation:

$$
X^{F}(t)=\int_{0}^{t} C(t, s)_{R L} D_{a+}^{1-\alpha} F(s) \mathrm{d} s,
$$

where $C(t, s)$ is the fundamental matrix of the system (5). Then from (25) after simple calculations and integrating by parts we obtain for $t \in \mathbb{R}_{+}$

$$
\begin{aligned}
X^{F}(t) & =\int_{0}^{t} C(t, s)_{R L} D_{0+}^{1-\alpha} F(s) \mathrm{d} s=I_{-1}(\Gamma(\alpha)) \int_{0}^{t} C(t, s)\left(\frac{\mathrm{d}}{\mathrm{d} s} \int_{0}^{s} I_{\alpha-1}(s-\eta) F(\eta) \mathrm{d} \eta\right) \mathrm{d} s \\
& =I_{-1}(\Gamma(\alpha)) \int_{0}^{t} C(t, s) \mathrm{d}_{s}\left(\int_{0}^{s} I_{\alpha-1}(s-\eta) F(\eta) \mathrm{d} \eta\right) \\
& \left.=I_{-1}(\Gamma(\alpha)) \int_{0}^{t} I_{\alpha-1}(t-\eta) F(\eta) \mathrm{d} \eta\right)-I_{-1}(\Gamma(\alpha)) \int_{0}^{t}\left(\int_{0}^{s} I_{\alpha-1}(s-\eta) F(\eta) \mathrm{d} \eta\right) \mathrm{d}_{s}(C(t, s))
\end{aligned}
$$

Then for the first addend in the right side of (26) using (12) we obtain that

$$
\begin{aligned}
& \left.\mid I_{-1}(\Gamma(\alpha)) \int_{0}^{t} I_{\alpha-1}(t-\eta) F(\eta) \mathrm{d} \eta\right)\left|\leq C_{0} \int_{0}^{t}\right| I_{\alpha-1}(t-\eta) \mid\|F(\eta)\| \mathrm{d} \eta=C_{0} \int_{0}^{t}(t-\eta)^{\alpha_{*}-1}\|F(\eta)\| \mathrm{d} \eta \\
& \text { and hence in virtue of (16) we obtain } \\
& \left.\| I_{-1}(\Gamma(\alpha)) \int_{0}^{t} I_{\alpha-1}(t-\eta) F(\eta) \mathrm{d} \eta\right)\left\|\leq C_{0} \int_{0}^{t}(t-\eta)^{\alpha_{*}-1}\right\| F(\eta)\left\|\mathrm{d} \eta \leq \alpha_{*}^{-1} C_{0} t^{\alpha_{*}}\right\| F(t) \| . \\
& \sup _{s \in[0, t]}\left|I_{-1}(\Gamma(\alpha)) \int_{0}^{t}\left(\int_{0}^{s} I_{\alpha-1}(s-\eta) F(\eta) \mathrm{d} \eta\right) \mathrm{d}_{s}(C(t, s))\right| \\
& \leq C_{0}\|\bar{C}(t, t)\| \sup _{s \in[0, t]}\left|\int_{0}^{s} I_{\alpha-1}(s-\eta) F(\eta) \mathrm{d} \eta\right| \leq C_{0}\|\bar{C}(t, t)\| \sup _{s \in[0, t]} \int_{0}^{s}\left|I_{\alpha-1}(s-\eta)\right|\|F(\eta)\| \mathrm{d} \eta \\
& =C_{0}\|F(t)\|\|\bar{C}(t, t)\| \sup _{s \in[0, t]} \int_{0}^{s}(t-\eta)^{\alpha_{*}-1} \mathrm{~d} \eta=\alpha_{*}^{-1} C_{0} t^{\alpha_{*}}\|F(t)\|\|\bar{C}(t, t)\| .
\end{aligned}
$$

Then the statement of the theorem follows from (27) and (28).

Theorem 5. Let $T \in \mathbb{R}_{+}$be an arbitrary fixed number and the following conditions are fulfilled:

1. The conditions $(S)$ hold.

2. $\|F(T)\|=0$.

3. The initial function $\Phi \in B V\left([-h, 0], \mathbb{R}^{n}\right) \cap \tilde{C}$ and its Lebesgue decomposition does not include a singular term. 
Then the corresponding unique solution $X_{\Phi}(t)$ of the IP (1), (2) for every $t \in J_{T}$ satisfies the estimation

$$
\left\|X_{\Phi}(t)\right\| \leq\left|\operatorname{Var}_{\eta \in[-h, 0]} \Phi(\eta)\right| \sup _{s \in[-h, 0]}\left\|T_{-h}(t, s)\right\|+|\Phi(-h)|\left\|T_{-h}(t-h)\right\| .
$$

Proof. According Theorem 9 in [31] the unique solution $X_{\Phi}(t)$ of the IP (1), (2) for every $t \in \mathbb{R}_{+}$has the following representation:

$$
X_{\Phi}(t)=\int_{-h}^{0} T_{-h}(t, s) \mathrm{d} \Phi(s)+T_{-h}(t,-h) \Phi(-h) .
$$

From (30) we obtain

$$
\begin{aligned}
\left\|X_{\Phi}(t)\right\| & \leq \int_{-h}^{0}\left\|T_{-h}(t, s)\right\| \mathrm{d}\left\|\operatorname{Var}_{\eta \in[-h, s]} \Phi(\eta)\right\|+|\Phi(-h)|\left\|T_{-h}(t,-h)\right\| \\
& \leq\left|\operatorname{Var}_{\eta \in[-h, 0]} \Phi(\eta)\right| \sup _{s \in[-h, 0]}\left\|T_{-h}(t, s)\right\|+|\Phi(-h)|\left\|T_{-h}(t-h)\right\|
\end{aligned}
$$

and from (31) it follows (29), which complete the proof.

Corollary 4. Let $T \in \mathbb{R}_{+}$be an arbitrary fixed number and the following conditions are fulfilled:

1. The conditions $(S)$ hold.

2. The initial functions $\Phi(t) \equiv \Phi_{0} \neq \mathbf{0}, \Phi_{0} \in \mathbb{R}^{n}$ for $t \in[-h, 0]$.

Then the corresponding unique solution $X_{\Phi}(t)$ of the IP (1), (2) for every $t \in J_{T}$ satisfies the estimation

$$
\left\|X_{\Phi}(t)\right\| \leq|\Phi(-h)|\left\|T_{-h}(t,-h)\right\|=\left|\Phi_{0}\right|\left\|T_{-h}(t,-h)\right\|
$$

Proof. According Theorem 9 in [31] the unique solution $X(t)$ of the IP (1), (2) for every $t \in \mathbb{R}_{+}$has the representation (30) and hence we obtain that $X_{\Phi}(t)=T_{-h}(t,-h) \Phi(-h)$ which completes the proof.

Corollary 5. Let $T \in \mathbb{R}_{+}$be an arbitrary fixed number and the following conditions are fulfilled:

1. The conditions $(S)$ hold.

2. The function $F(t) \in L_{1}^{\text {loc }}\left(\mathbb{R}_{+}, \mathbb{R}^{n}\right)$ and is locally bounded.

3. The initial function $\Phi \in B V\left([-h, 0], \mathbb{R}^{n}\right) \cap \tilde{C}$ and its Lebesgue decomposition does not include a singular term.

Then the corresponding unique solution $X_{\Phi}^{F}(t)$ of the IP (1), (2) for every $t \in J_{T}$ satisfies the estimation

$$
\begin{aligned}
\left\|X_{\Phi}^{F}(t)\right\| & \leq\left|\operatorname{Var}_{\eta \in[-h, 0]} \Phi(\eta)\right| \sup _{s \in[-h, 0]}\left\|T_{-h}(t, s)\right\|+|\Phi(-h)|\left\|T_{-h}(t-h)\right\| \\
& +\alpha_{*}^{-1} t^{\alpha_{*}} C_{0}\|F(t)\|(1+\|\bar{C}(t, t)\|)
\end{aligned}
$$

Proof. Using the superposition principle, i.e., $X_{\Phi}^{F}(t)=X_{\Phi}(t)+X^{F}(t)$ we obtain that the estimation (33) follows immediately from Theorems 4 and 5.

Remark 3. It is clear that if $\|\Phi\|\|F(T)\|>0$, then (33) can be rewritten in the form

$$
\begin{aligned}
\left\|X_{\Phi}^{F}(t)\right\| & \leq \max \left(\|\Phi\|,\left|\operatorname{Var}_{\eta \in[-h, 0]} \Phi(\eta)\right|\right)\left[\sup _{s \in[-h, 0]}\left\|T_{-h}(t, s)\right\|+\left\|T_{-h}(t-h)\right\|\right. \\
& \left.+\alpha_{*}^{-1} t^{\alpha_{*}} C_{0} C_{\Phi}(1+\|\bar{C}(t, t)\|)\right]
\end{aligned}
$$


The next theorem establishes explicit bounds for the matrix functions involved in (33) and (34), which allows obtaining a new form of these estimations more convenient for practical computer calculations.

Theorem 6. Let $T \in \mathbb{R}_{+}$be an arbitrary fixed number and the following conditions are fulfilled:

1. The conditions $(S)$ hold.

2. The function $F(t) \in L_{1}^{\text {loc }}\left(\mathbb{R}_{+}, \mathbb{R}^{n}\right)$ and is locally bounded and $\|\Phi\|\|F(T)\|>0$.

3. The initial function $\Phi \in B V\left([-h, 0], \mathbb{R}^{n}\right) \cap \tilde{C}$ and its Lebesgue decomposition does not include a singular term.

Then the corresponding unique solution $X_{\Phi}^{F}(t)$ of the IP (1), (2) for every $t \in J_{T}$ satisfies the estimation

$$
\begin{aligned}
\left\|X_{\Phi}^{F}(t)\right\| & \leq\left(\left|\operatorname{Var}_{\eta \in[-h, 0]} \Phi(\eta)\right|+|\Phi(-h)|\right) E_{\alpha}\left(\|\bar{U}(t, 0)\| C_{0} \Gamma\left(\alpha_{*}\right) t^{\alpha_{*}}\right) \\
& +\alpha_{*}^{-1} t^{\alpha_{*}} C_{0}\|F(t)\|\left(1+2 E_{\alpha}\left(\|\bar{U}(t, 0)\| C_{0} \Gamma\left(\alpha_{*}\right) t^{\alpha_{*}}\right)\right)
\end{aligned}
$$

Proof. From (7) it follows that $\left\|\Phi_{1}(t, s)\right\|=1, t \in(-\infty, s]$ and $\left\|\Phi_{2}(t, s)\right\|=1, s \in$ $[-h, 0], t \in[s, 0]$.

Let $s \in \overline{\mathbb{R}}_{+}$be an arbitrary fixed number and $C(t, s)$ is the solution for $t \in(s, \infty)$ of the (7) with initial condition $C(t, s)=\Phi_{1}(t, s), t \in(-\infty, s]$. Then from (7), (8) it follows that

$$
C(t, s)=I+I_{-1}(\Gamma(\alpha)) \int_{s}^{t} I_{\alpha-1}(t-\eta) \int_{-h}^{0}\left[\mathrm{~d}_{\theta} U(\eta, \theta)\right] C(\eta+\theta, s) \mathrm{d} \eta
$$

and respectively for $s \in[-h, 0], t \in \mathbb{R}_{+}$we have that

$$
T_{-h}(t, s)=I+I_{-1}(\Gamma(\alpha)) \int_{s}^{t} I_{\alpha-1}(t-\eta) \int_{-h}^{0}\left[\mathrm{~d}_{\theta} U(\eta, \theta)\right] T_{-h}(\eta+\theta, s) \mathrm{d} \eta
$$

where $T_{-h}(t, s)=\Phi_{2}(t, s), t \in(-\infty, 0]$.

For arbitrary fixed $s \in \overline{\mathbb{R}}_{+}$, since $\|C(t, s)\|$ is nonnegative and nondecreasing in $t$ from the first system (36) and (16) we obtain that

$$
\begin{aligned}
\|C(t, s)\| & =\sup _{\xi \in[0, t]}\left|C(\xi, s) \leq 1+C_{0} \sup _{\xi \in[0, t]} \int_{s}^{\xi}\right| I_{\alpha-1}(\xi-\eta)|| \int_{-h}^{0}\left[\mathrm{~d}_{\theta} U(\eta, \theta)\right] C(\eta+\theta, s) \mid \mathrm{d} \eta \\
& \leq 1+C_{0}\|\bar{U}(t, 0)\| \sup _{\xi \in[0, t]} \int_{0}^{\xi}(\xi-\eta)^{\alpha_{*}-1} \sup _{\eta+\theta \in[-h, \xi]}|C(\eta+\theta, s)| \mathrm{d} \eta \\
& \leq 1+C_{0}\|\bar{U}(t, 0)\| \sup _{\xi \in[0, t]} \int_{0}^{\xi}(\xi-\eta)^{\alpha_{*}-1}\|C(\eta, s)\| \mathrm{d} \eta \\
& \leq 1+C_{0}\|\bar{U}(t, 0)\| \int_{0}^{t}(t-\eta)^{\alpha_{*}-1}\|C(\eta, s)\| \mathrm{d} \eta
\end{aligned}
$$

and then in virtue of Corollary 1 we have that

$$
\|C(t, s)\| \leq E_{\alpha}\left(\|\bar{U}(t, 0)\| C_{0} \Gamma\left(\alpha_{*}\right) t^{\alpha_{*}}\right) \leq E_{\alpha}\left(\|\bar{U}(T, 0)\| C_{0} \Gamma\left(\alpha_{*}\right) T^{\alpha_{*}}\right), s \in \overline{\mathbb{R}}_{+}
$$


Analogical way when $T_{-h}(t, s)$ is a solution of the (8) with initial condition $T_{-h}(t, s)=$ $\Phi_{2}(t, s), t \in(-\infty, 0]$ and since $\left\|T_{-h}(t, s)\right\|$ is nonnegative and nondecreasing in $t$ from (16) and (37) we obtain

$$
\begin{aligned}
\left\|T_{-h}(t, s)\right\| & \leq 1+C_{0} \sup _{\xi \in[0, t]} \int_{s}^{\xi}\left|I_{\alpha-1}(\xi-\eta)\right|\left|\int_{-h}^{0}\left[\mathrm{~d}_{\theta} U(\eta, \theta)\right] T_{-h}(\eta+\theta, s)\right| \mathrm{d} \eta \\
& \leq 1+C_{0}\|\bar{U}(t, 0)\| \sup _{\xi \in[0, t]} \int_{0}^{t}(\xi-\eta)^{\alpha_{*}-1} \sup _{\theta \in[-h, 0]}\left|T_{-h}(\eta+\theta, s)\right| \mathrm{d} \eta \\
& \leq 1+C_{0}\|\bar{U}(t, 0)\| \sup _{\xi \in[0, t]} \int_{0}^{\zeta}(\xi-\eta)^{\alpha_{*}-1}\left\|T_{-h}(\eta, s)\right\| \mathrm{d} \eta \\
& \leq 1+C_{0}\|\bar{U}(t, 0)\| \int_{0}^{t}(t-\eta)^{\alpha_{*}-1}\left\|T_{-h}(\eta, s)\right\| \mathrm{d} \eta
\end{aligned}
$$

and hence in virtue of Corollary 1 we have

$$
\mid T_{-h}(t, s) \| \leq E_{\alpha}\left(\|\bar{U}(t, 0)\| C_{0} \Gamma\left(\alpha_{*}\right) t^{\alpha_{*}}\right) \leq E_{\alpha}\left(\|\bar{U}(T, 0)\| C_{0} \Gamma\left(\alpha_{*}\right) T^{\alpha_{*}}\right), s \in[-h, 0] .
$$

Since for fixed $t$ the matrix function $\|\bar{C}(t, s)\|$ is nondecreasing for $s \in[0, T]$, then taking into account (39) and (40) we have that

$$
\|\bar{C}(T, T)\|=\|C(T, T)-C(T, 0)\| \leq\|C(T, T)\|+\|C(T, 0)\| \leq 2 E_{\alpha}\left(\|\bar{U}(T, 0)\| C_{0} \Gamma\left(\alpha_{*}\right) T^{\alpha_{*}}\right) .
$$

Then from (40) and (41) we obtain that for every $t \in J_{T}$ the estimation (35) holds.

Remark 4. Please note that if $\|\Phi\|\|F(T)\|>0$, then (35) can be rewritten in the form

$$
\begin{aligned}
& \left\|X_{\Phi}^{F}(t)\right\| \leq \max \left(\|\Phi\|,\left|\operatorname{Var}_{\eta \in[-h, 0]} \Phi(\eta)\right|\right) \\
& \quad\left[2 E_{\alpha}\left(\|\bar{U}(t, 0)\| C_{0} \Gamma\left(\alpha_{*}\right) t^{\alpha_{*}}\right)+\alpha_{*}^{-1} t^{\alpha_{*}} C_{0} C_{\Phi}\left(1+2 E_{\alpha}\left(\|\bar{U}(t, 0)\| C_{0} \Gamma\left(\alpha_{*}\right) t^{\alpha_{*}}\right)\right]\right.
\end{aligned}
$$

\section{Finite-Time Stability Results}

In this section, we study the finite-time stability (FTS) properties of the system (1), with the initial condition (2) as an application of the different a priori estimations obtained in Sections 4 and 5. In addition, we will study these properties for different types initial functions. A special attention obtains the case when $\|\Phi\|=0$ too.

First, we start with the homogeneous case, i.e., the IP (4), (2).

Theorem 7. Let $T \in \mathbb{R}_{+}$be an arbitrary fixed number and the following conditions are fulfilled:

1. The conditions (S) hold and $F(t) \equiv \mathbf{0}$ for $t \in J_{T}$.

2. There exist numbers $\varepsilon \geq \delta>0$ such that the following inequality holds

$$
\delta E_{\alpha}\left(\|\bar{U}(T, 0)\| C_{0} \Gamma\left(\alpha_{*}\right) T^{\alpha_{*}}\right) \leq \varepsilon
$$

Then for every initial function $\Phi \in \tilde{C}$ with $\|\Phi\|<\delta$ the corresponding unique solution $X(t)$ of the IP (1), (2) (in this case this is IP (4), (2)) is finite-time stable with respect to $\left\{0, J_{T}, \delta, \varepsilon, h\right\}$.

Proof. Let $\Phi \in \tilde{C}$ with $\|\Phi\|<\delta$ be an arbitrary initial function. Then if $\max (\|X(T)\|,\|\Phi\|)=\|\Phi\|$ then the statement of the theorem holds. The nontrivial case obviously is when $\max (\|X(T)\|,\|\Phi\|)>\|\Phi\|$. In this case from condition 1 it follows that Corollary 2 holds and from (18) for $t \in J_{T}$ we obtain that

$$
\|X(t)\| \leq\|\Phi\| E_{\alpha}\left(\|\bar{U}(t, 0)\| C_{0} \Gamma\left(\alpha_{*}\right) t^{\alpha_{*}}\right)
$$


and hence from (43) and (44) it follows that

$$
\|X(t)\|<\|\Phi\| E_{\alpha}\left(\|\bar{U}(t, 0)\| C_{0} \Gamma\left(\alpha_{*}\right) t^{\alpha_{*}}\right) \leq \delta E_{\alpha}\left(\|\bar{U}(T, 0)\| C_{0} \Gamma\left(\alpha_{*}\right) T^{\alpha_{*}}\right) \leq \varepsilon
$$

which completes the proof.

The next theorem considers a special nonhomogeneous case of the system (1) when $\|\Phi\|=0$.

Theorem 8. Let the following conditions be fulfilled:

1. The conditions of Theorem 2 hold and $\|\Phi\|=0$.

2. There exist numbers $\varepsilon \geq \delta>0$ such that if $\|F(T)\|<\delta$ then the following inequality holds

$$
\delta \alpha_{*}^{-1} C_{0} T^{\alpha_{*}} E_{\alpha}\left(\|\bar{U}(t, 0)\| C_{0} \Gamma\left(\alpha_{*}\right) T^{\alpha_{*}}\right) \leq \varepsilon
$$

Then the corresponding unique solution $X(t)$ of the IP (1), (2) is finite-time stable with respect to $\left\{0, J_{T}, \delta, \varepsilon, h\right\}$.

Proof. Let us consider the case when $\max (\|X(T)\|,\|\Phi\|)>\|\Phi\|$. Since Corollary 3 holds, from (19) and (45) for $t \in J_{T}$ it follows that

$$
\begin{aligned}
\|X(t)\| & \leq \alpha_{*}^{-1} C_{0} t^{\alpha_{*}}\|F(t)\| E_{\alpha}\left(\|\bar{U}(t, 0)\| C_{0} \Gamma\left(\alpha_{*}\right) t^{\alpha_{*}}\right) \\
& \leq \delta \alpha_{*}^{-1} C_{0} T^{\alpha_{*}} E_{\alpha}\left(\|\bar{U}(t, 0)\| C_{0} \Gamma\left(\alpha_{*}\right) T^{\alpha_{*}}\right) \leq \varepsilon
\end{aligned}
$$

Thus, from (46) it follows that the corresponding unique solution $X(t)$ of the IP (1), (2) is finite-time stable with respect to $\left\{0, J_{T}, \delta, \varepsilon, h\right\}$ for every locally bounded $F(t) \in$ $L_{1}^{\text {loc }}\left(\mathbb{R}_{+}, \mathbb{R}^{n}\right)$.

Theorem 9. Let the following conditions be fulfilled:

1. The conditions of Theorem 2 hold and $\|\Phi\|>0$.

2. There exist numbers $\varepsilon \geq \delta>0$ such that if $\|\Phi\|<\delta$ then the following inequality holds

$$
\delta\left(1+\alpha_{*}^{-1} C_{0} C_{\Phi} T^{\alpha_{*}}\right) E_{\alpha}\left(\|\bar{U}(T, 0)\| C_{0} \Gamma\left(\alpha_{*}\right) T^{\alpha_{*}}\right) \leq \varepsilon
$$

Then for every initial function $\Phi \in \tilde{C}$ with $\|\Phi\| \in(0, \delta)$ the corresponding unique solution $X(t)$ of the IP (1), (2) is finite-time stable with respect to $\left\{0, J_{T}, \delta, \varepsilon, h\right\}$.

Proof. Let $\Phi \in \tilde{C}$ with $\|\Phi\| \in(0, \delta)$ be an arbitrary initial function and assume that $\max (\|X(T)\|,\|\Phi\|)>\|\Phi\|$. Then since Theorem 2 holds, from (23) and (47) for $t \in J_{T}$ it follows that

$$
\begin{aligned}
\|X(t)\| & \leq\|\Phi\|\left(1+\alpha_{*}^{-1} C_{0} C_{\Phi} t^{\alpha_{*}}\right) E_{\alpha}\left(\|\bar{U}(t, 0)\| C_{0} \Gamma\left(\alpha_{*}\right) t^{\alpha_{*}}\right) \\
& \leq \delta\left(1+\alpha_{*}^{-1} C_{0} C_{\Phi} t^{\alpha_{*}}\right) E_{\alpha}\left(\|\bar{U}(T, 0)\| C_{0} \Gamma\left(\alpha_{*}\right) T^{\alpha_{*}}\right) \leq \varepsilon
\end{aligned}
$$

Thus, from (48) it follows that for every initial function $\Phi \in \tilde{C}$ with $\|\Phi\| \in(0, \delta)$ the corresponding unique solution $X(t)$ of the IP $(1),(2)$ is finite-time stable with respect to $\left\{0, J_{T}, \delta, \varepsilon, h\right\}$.

Below we present FTS results based on estimations obtained via different kind integral representations of the solutions and superposition principle.

Theorem 10. Let the following conditions be fulfilled:

1. The conditions of Theorem 4 hold.

2. There exist numbers $\varepsilon \geq \delta>0$ such that if $\|F(T)\|<\delta$ then the following inequality holds

$$
\delta \alpha_{*}^{-1} C_{0} T^{\alpha_{*}}(1+\|\bar{C}(T, T)\|) \leq \varepsilon
$$


Then for the initial function $\Phi \in \tilde{C}$ with $\|\Phi\|=0$ and locally bounded function $F(t) \in$ $L_{1}^{\text {loc }}\left(\mathbb{R}_{+}, \mathbb{R}^{n}\right)$ with $\|F(T)\|<\delta$ the corresponding unique solution $X(t)$ of the IP (1), (2) is finite-time stable with respect to $\left\{0, J_{T}, \delta, \varepsilon, h\right\}$.

Proof. Theorem 4 implies that for each $t \in J_{T}$ the inequality (24) holds and then from (24) and (49) for every $t \in J_{T}$ it follows that

$\|X(t)\| \leq \alpha_{*}^{-1} C_{0} t^{\alpha_{*}}\|F(t)\|(1+\|\bar{C}(t, t)\|)<\delta \alpha_{*}^{-1} C_{0} t^{\alpha_{*}}(1+\|\bar{C}(t, t)\|) \leq \delta \alpha_{*}^{-1} C_{0} T^{\alpha_{*}}(1+\|\bar{C}(T, T)\|) \leq \varepsilon$

which completes the proof.

Theorem 11. Let the following conditions be fulfilled:

1. The conditions of Theorem 5 hold.

2. There exist numbers $\varepsilon \geq \delta>0$ such that if $\max \left(|\Phi(-h)|,\left|\operatorname{Var}_{\eta \in[-h, 0]} \Phi(\eta)\right|\right)<\delta$ then the following inequality holds

$$
\delta\left(\sup _{s \in[-h, 0]}\left\|T_{-h}(T, s)\right\|+\left\|T_{-h}(T,-h)\right\|\right) \leq \varepsilon
$$

Then the corresponding unique solution $X(t)$ of the IP (1), (2) is finite-time stable with respect to $\left\{0, J_{T}, \delta, \varepsilon, h\right\}$.

Proof. Theorem 5 implies that for each $t \in J_{T}$ the inequality (29) holds and then from (29) and (50) same way as above for every $t \in J_{T}$ we obtain that

$$
\begin{aligned}
\|X(t)\| & \leq\left|\operatorname{Var}_{\eta \in[-h, 0]} \Phi(\eta)\right| \sup _{s \in[-h, 0]}\left\|T_{-h}(t, s)\right\|+\|\Phi(-h)\|\left\|T_{-h}(t,-h)\right\| \\
& \leq \delta\left(\sup _{s \in[-h, 0]}\left\|T_{-h}(T, s)\right\|+\left\|T_{-h}(T,-h)\right\|\right) \leq \varepsilon
\end{aligned}
$$

and hence the corresponding unique solution $X(t)$ of the IP (1), (2) is finite-time stable with respect to $\left\{0, J_{T}, \delta, \varepsilon, h\right\}$.

Corollary 6. Let the following conditions be fulfilled:

1. The conditions of Corollary 4 , hold.

2. There exist numbers $\varepsilon \geq \delta>0$ such that if $|\Phi(-h)|<\delta$ then the following inequality holds

$$
\delta\left\|T_{-h}(T,-h)\right\| \leq \varepsilon
$$

Then the corresponding unique solution $X(t)$ of the IP (1), (2) is finite-time stable with respect to $\left\{0, J_{T}, \delta, \varepsilon, h\right\}$.

Proof. Since $\|\Phi\|=\left|\Phi_{0}\right|=|\Phi(-h)|<\delta$ then using (32) and (51)we obtain

$$
\|X(t)\| \leq|\Phi(-h)|\left\|T_{-h}(t,-h)\right\|=\left|\Phi_{0}\right|\left\|T_{-h}(t,-h)\right\| \leq \delta\left\|T_{-h}(t,-h)\right\| \leq \varepsilon
$$

and then the result follows from Theorem 11.

Remark 5. The FTS results obtained in Theorem 11 and Corollary 6 are new even in the cases considered in [25] when the initial function $\Phi \in C^{1}\left([-h, 0], \mathbb{R}^{n}\right)$. Our results are more accurate not only in the case when the initial function $\Phi \in B V\left([-h, 0], \mathbb{R}^{n}\right)$ has finite set of jump points $S_{\Phi} \neq \varnothing$, (i.e., $\Phi$ is not continuous), but also when $\Phi$ is continuous.

We illustrate this fact with two simple examples:

Let $\Phi(-h)=(0.75,0)^{T}, \Phi(t)=(1,0)^{T}, t \in(-h, 0]$. Then $|\Phi(-h)|=0.75,\|\Phi\|=$ $1,\left|\operatorname{Var}_{\eta \in[-h, 0]} \Phi(\eta)\right|=0.25$ and $\max \left(\left|\operatorname{Var}_{\eta \in[-h, 0]} \Phi(\eta)\right|,|\Phi(-h)|\right)=|\Phi(-h)|=0.75<$ $\|\Phi\|=1$. 
Let $h=1$ and $\Phi(t)=(0.4 t+1,0)^{T}, t \in(-1,0], \Phi(-1)=(0.6,0)^{T},\left|\operatorname{Var}_{\eta \in[-1,0]} \Phi(\eta)\right|=$ 0.6, $\|\Phi\|=1$ and hence $\max \left(\left|\operatorname{Var}_{\eta \in[-1,0]} \Phi(\eta)\right|,|\Phi(-1)|\right)=0.6<\|\Phi\|=1$.

These examples show, that we can establish FTS in some cases, where the conditions presented in [25] are not directly applicable.

Remark 6. The FTS result for the general case $\|\Phi\|\|F(T)\|>0$ needs some preliminary comments.

It is clear that the estimations (32) and (33) will be essentially used, but to obtain a practical applicable estimation we need to solve (clarify) two problems:

(a) First, we need to clarify which impact is leading for the process, the impact hereditary of the process expressed by $\|\Phi\|$, the impact of the outer perturbations expressed by $\|F(T)\|$, or the complex of both factors expressed by the ratio $C_{\Phi}=\|\Phi\|^{-1}\|F(T)\|$.

(b) As second, an explicit estimation is needed in the general case for the fundamental matrix $C(t, s)$ as well as the matrix $T_{-h}(t, s)$ too.

Concerning point (a), it is clear that a reasonable response can be given only on the basis of real empirical data from the process which is described by the mathematical model. From a mathematical point of view, as was mentioned above by the construction of the proofs, we must limit the impact of $\|\Phi\|$ and $\|F(T)\|$ to linear or no more than power-law growth as in the right side of the estimation (23) and avoid the high nonlinear impact of $C_{\Phi}=\|\Phi\|^{-1}\|F(T)\|$ if it is involved as an argument in the Mittag-Leffler function $E_{\alpha}(\cdot)$ in (20).

About (b) it is possible to obtain the needed estimations in the general case, for example we can use the estimations obtained in the previous sections.

Theorem 12. Let $T \in \mathbb{R}_{+}$be an arbitrary fixed number and the following conditions are fulfilled:

1. The conditions $(S)$ hold.

2. The function $F(t) \in L_{1}^{\text {loc }}\left(\mathbb{R}_{+}, \mathbb{R}^{n}\right)$ and is locally bounded.

3. The initial function $\Phi \in B V\left([-h, 0], \mathbb{R}^{n}\right) \cap \tilde{C}$ and its Lebesgue decomposition does not include a singular term.

4. $\|\Phi\|\|F(T)\|>0$ and there exist numbers $\varepsilon \geq \delta>0$ such that if $\max (|\Phi(-h)|$, $\left.\left|\operatorname{Var}_{\eta \in[-h, 0]} \Phi(\eta)\right|\right)<\delta$ then the following inequality holds

$$
\delta\left[\sup _{s \in[-h, 0]}\left\|T_{-h}(T, s)\right\|+\left\|T_{-h}(T,-h)\right\|+\alpha_{*}^{-1} T^{\alpha_{*}} C_{0} C_{\Phi}(1+\|\bar{C}(T, T)\|)\right] \leq \varepsilon
$$

Then the corresponding unique solution $X(t)$ of the IP (1), (2) for every $t \in J_{T}$ is finite-time stable with respect to $\left\{0, J_{T}, \delta, \varepsilon, h\right\}$.

Proof. Condition 4 of the theorem implies that the estimate (34) holds. Then from (34) and (52) for every $t \in J_{T}$ it follows

$$
\begin{aligned}
\|X(t)\| & \leq\left|\operatorname{Var}_{\eta \in[-h, 0]} \Phi(\eta)\right| \sup _{s \in[-h, 0]}\left\|T_{-h}(t, s)\right\|+|\Phi(-h)|\left\|T_{-h}(t,-h)\right\| \\
& +\alpha_{*}^{-1} t^{\alpha_{*}} C_{0}\|F(t)\|(1+\|\bar{C}(t, t)\|) \\
& \leq \delta\left[\sup _{s \in[-h, 0]}\left\|T_{-h}(t, s)\right\|+\left\|T_{-h}(t,-h)\right\|+\alpha_{*}^{-1} t^{\alpha_{*}} C_{0} C_{\Phi}(1+\|\bar{C}(t, t)\|)\right] \\
& \leq \delta\left[\sup _{s \in[-h, 0]}\left\|T_{-h}(T, s)\right\|+\left\|T_{-h}(T,-h)\right\|+\alpha_{*}^{-1} t^{\alpha_{*}} C_{0} C_{\Phi}(1+\|\bar{C}(T, T)\|)\right] \leq \varepsilon
\end{aligned}
$$

which completes the proof.

Corollary 7. Let $T \in \mathbb{R}_{+}$be an arbitrary fixed number and the following conditions are fulfilled:

1. The conditions $(S)$ hold.

2. The function $F(t) \in L_{1}^{\text {loc }}\left(\mathbb{R}_{+}, \mathbb{R}^{n}\right)$ and is locally bounded.

3. The initial function $\Phi \in B V\left([-h, 0], \mathbb{R}^{n}\right) \cap \tilde{C}$ and its Lebesgue decomposition does not include a singular term. 
4. $\|\Phi\|\|F(T)\|>0$ and there exist numbers $\varepsilon \geq \delta>0$ such that if $\max (|\Phi(-h)|$, $\left.\left|\operatorname{Var}_{\eta \in[-h, 0]} \Phi(\eta)\right|\right)<\delta$ then the following inequality holds

$$
\delta\left[2 E_{\alpha}\left(\|\bar{U}(T, 0)\| C_{0} \Gamma\left(\alpha_{*}\right) T^{\alpha_{*}}\right)+\alpha_{*}^{-1} T^{\alpha_{*}} C_{0} C_{\Phi}\left(1+2 E_{\alpha}\left(\|\bar{U}(T, 0)\| C_{0} \Gamma\left(\alpha_{*}\right) T^{\alpha_{*}}\right)\right] \leq \varepsilon\right.
$$

Then the corresponding unique solution $X(t)$ of the IP (1), (2) for every $t \in J_{T}$ is finite-time stable with respect to $\left\{0, J_{T}, \delta, \varepsilon, h\right\}$.

Proof. The statement follows from Theorem 12 and Theorem 6.

\section{Examples and Comments}

Remark 7. From a practical point of view, it is important to establish a sharp upper bound of the constant $\alpha_{*}^{-1} C_{0}$ appearing in all estimates except (29) and answer the question does the constant $\alpha_{*}^{-1} C_{0}$ attain its upper bound.

Let us consider the case when $\alpha_{*}=\alpha_{M}$. Then we have that $\alpha_{*}^{-1} C_{0}=\alpha_{M}^{-1} C_{0}=\Gamma^{-1}(1+$ $\left.\alpha_{M}\right) \leq \Gamma^{-1}\left(z_{\min }\right)$. Thus if $\alpha_{M}=z_{\text {min }}-1$ then $\alpha_{*}^{-1} C_{0}$ attains its upper bound, namely $\alpha_{*}^{-1} C_{0}=$ $\Gamma^{-1}\left(z_{\min }\right) \approx 1.1279$. Please note that in the partial case when all orders of the differentiation coincide (i.e., $\alpha_{1}=\cdots=\alpha_{n}=\alpha$ ) then all estimates can be essentially simplified. For example in this case we have that $\alpha_{*}^{-1} C_{0}=\Gamma^{-1}(1+\alpha) \leq \Gamma^{-1}\left(z_{\min }\right)$ and $C_{0} \Gamma \alpha_{*}=1$.

Remark 8. First, it must be noted that in the commented works are used different norms. In the works $[24,25]$ the so-called 1-norm is used (i.e., for $W=\left\{w_{i j}\right\}_{i, j \in\langle n\rangle} \in \mathbb{R}^{n \times n}$ the matrix norm $\left.|W|=\max _{j \in\langle n\rangle} \sum_{i=1}^{n}\left|w_{i j}\right|\right)$ while in $[26,27]$ is used the spectral norm as well as in our work. A direct comparison shows that the condition (43) in our work based on the estimate (18) is more accurate in compare with the condition (9) in Theorem 4.1 [24] proved via the integral representation approach and condition (16) in Theorem 3.2 in [27] proved by Gronwall's approach, even in the partial cases considered in these works.

Please note that for the partial case when $\Phi$ is a constant both conditions (43) and (9) in [24] coincide. In this case the same results can be established by using (50) obtained via the integral representation (30). In the homogeneous case $(\gamma=0)$ of the considered in [26] partial cases of the system (4) (variable matrices and one variable delay), our condition (43) coincides with condition (5) of Theorem 1 in [26] proved by Gronwall's approach.

Below on the base of the considered in the work [24] example we will establish that generally speaking the results obtained via the integral representation approach can be more accurate in comparison with these obtained via the Gronwall's approach but the results depend essentially from the norm choice and from the constructions of their proofs.

Example 1. [24] Consider

$$
\begin{cases}D_{0+}^{\alpha} X(t)=A X(t-\sigma), & t>0 \\ X(t)=\Phi(t), & t \in[-\sigma, 0]\end{cases}
$$

where $A=\left(\begin{array}{cc}0.2 & 0 \\ 0 & 0.8\end{array}\right), \alpha=0.2, \sigma=0.2, T=0.8, \Phi(t)=(0.1,0.2)^{T}$.

The system (54) is a partial case of (4) in the case when: $n=2, \alpha_{1}=\alpha_{2}=\alpha=$ $0.2, U_{A C}(t, \theta)=U_{S}(t, \theta) \equiv \Theta, U_{J}(t, \theta)=A^{1} H(\theta+\sigma), A^{1}=A, A^{0}=\Theta, \sigma=h=$ $0.2,\|\bar{U}(T, 0)\|=\|A\|_{1}=\|A\|_{2}=0.8\|\Phi\|_{2}=|\Phi(-0.2)|=0.2236$.

Using system Wolfram Mathematica, we obtain $|\Phi(-0.2)| E_{0.2}\left(0.8 * 0.8^{0.2}\right)=0.2236 *$ $1.25913=0.9292$ and hence (54) is finite-time stable with respect to $\left\{0, J_{T}, \delta, \varepsilon, \sigma\right\}$ for $\varepsilon \geq 0.9292$.

The compared results are given in Table 1 below: 
Table 1. Compared Results.

\begin{tabular}{lccccc}
\hline Theorem/Work & $\|\boldsymbol{\Phi}\|$ & $\sigma=h$ & $\delta$ & $\|\boldsymbol{X}(\boldsymbol{t})\|$ & $\boldsymbol{F T S}$ \\
\hline Th. 4.1 in [24] & 0.3 & 0.2 & 0.31 & 1.2882 & Yes \\
\hline Th. 4.2 in [24] & 0.3 & 0.2 & 0.31 & 2.0586 & Yes \\
\hline Theorem 7 & 0.2236 & 0.2 & 0.2237 & 0.9292 & Yes \\
\hline Corollary 6 & 0.2236 & 0.2 & 0.2237 & 0.9292 & Yes \\
\hline Th. 1 $(\gamma=0)[26]$ & 0.2236 & 0.2 & 0.2237 & 0.9292 & Yes \\
\hline
\end{tabular}

Remark 9. Please note that the results essentially depend from the used norm and we can show that the spectral norm bring some advantages.

For example for the initial function $\Phi(t)=\left(\begin{array}{c}0.222 \\ 0.2\end{array}\right),\|\Phi\|_{1}=0.422,\|\Phi\|_{2}=0.299$ and for $\varepsilon=1.2882$ concerning the spectral norm (54) is FTS, which cannot be established using the 1-norm.

The same remark is also true concerning the matrix $A=\left(\begin{array}{cc}0.2 & 0 \\ 0 & 0.8\end{array}\right)$. Since $A$ is a diagonal matrix then $\|A\|_{1}=\|A\|_{2}=0.8$ but if for example we have $\bar{A}=\left(\begin{array}{cc}0.2 & 0.3 \\ 0 & 0.8\end{array}\right)$ then $\|A\|_{1}=1.1$, but $\|A\|_{2}=0.85742$ and then if we use some of the proved estimations, without direct calculation which for example we present, then the differences between the estimations will increase.

One direct calculation via the integral representation established in [24] for sharp upper bounds for the 1-norm and the spectral norm of the state vector for $T=0.8$ give us $\| X\left(0.8 \|_{1}=0.95702\right.$ and $\| X\left(0.8 \|_{2}=0.84059\right.$. Namely the solution of (54) according Theorem 3.2 in [24] has the following representation $X(t)=\boldsymbol{E}_{\sigma}^{B t^{\alpha}} \Phi(-\sigma)$, where $\Phi$ is a constant vector and $\boldsymbol{E}_{\sigma}^{B t^{\alpha}}=I+$ $\sum_{k=1}^{\infty} A^{k} \frac{(t-(k-1) \sigma)^{k \alpha}}{\Gamma(\alpha k+1)} H(k \sigma-t), t \in \overline{\mathbb{R}}_{+}, \boldsymbol{E}^{B t_{\theta}^{\alpha}}=\Theta$ for $t<-\sigma$ and $\boldsymbol{E}_{\sigma}^{B t^{\alpha}}=I$ for $-\sigma \leq t \leq 0$ is the introduced in the same work delayed matrix with Mittag-Leffler functions. For the values in the example above we have that

$$
X(t)=E_{0.2}^{A t^{0.2}} \Phi(-0.2)=\left(\begin{array}{cc}
E_{0.2}^{0.2 t^{0.2}} & 0 \\
0 & E_{0.2}^{0.8 t^{0.2}}
\end{array}\right)\left(\begin{array}{l}
0.1 \\
0.2
\end{array}\right)
$$

where the matrix entries are standard scalar Mittag-Leffler functions.

Calculating by system Wolfram Mathematica we obtain

$$
X(0.8)=\left(\begin{array}{cc}
1.25913 & 0 \\
0 & 4.15554
\end{array}\right)\left(\begin{array}{l}
0.1 \\
0.2
\end{array}\right)=\left(\begin{array}{c}
0.125913 \\
0.8311
\end{array}\right)
$$

and hence $\|X(0.8)\|_{1}=0.95702$ and $\|X(0.8)\|_{2}=0.84059$.

Finally, we note that the integral representation of the solution of (54) proved in Theorem 3.2 in [24] for the case when $\Phi \in C^{1}\left([-\tau, 0], \mathbb{R}^{n}\right.$ is partial case from the integral representation (4.7) in [31] proved for $\Phi \in B V\left([-\tau, 0], \mathbb{R}^{n}\right)$. For the system (54) the both presentations coincide when $\Phi \in A C\left([-\tau, 0], \mathbb{R}^{n}\right)$.

Analogically as in the homogeneous case consider one partial case of the IP (1), (2) as follows:

Example 2. Consider

$$
\begin{cases}D_{0+}^{\alpha} X(t)=A_{0}(t) X(t)+A_{1}(t) X(t-\sigma(t))+f(t, X(t)), & t>0 \\ X(t)=\Phi(t), & t \in[-\sigma, 0]\end{cases}
$$

The system (55) is considered in [25] in the case when $f \in C\left(\overline{\mathbb{R}}_{+} \times \mathbb{R}^{n}, \mathbb{R}^{n}\right), A_{0}(t) \equiv \Theta, A_{1}(t) \equiv$ $B \in \mathbb{R}^{n \times n}, \sigma(t) \equiv \sigma$ for $t \in \overline{\mathbb{R}}_{+}$. In the same work an example is given to clear the applicability of 
the theoretical results by using the following data: $\alpha_{1}=\alpha_{2}=\alpha=0.6, \sigma=0.2, T=0.6, \Phi(t)=$ $(t, 2 t)^{T}, \omega(t)=\psi(t)=2 t^{2}, A^{0}=\Theta, A_{1}=\left(\begin{array}{cc}0.3 & 0 \\ 0 & 0.5\end{array}\right)$ and $\|f(t, Y)\|_{1} \leq \omega(t)$ for all $t \in[0, T]$ and $Y \in \mathbb{R}^{n}$.

Let define $\|F(t)\|_{1}=\sup _{Y \in \in^{\sim n}}\|f(t, Y)\|_{1} \leq 2 t^{2}$. We will use the estimation (47) and then apply Theorem 9. In our notations we have: $\|\bar{U}(T, 0)\|=0.5,\|F(T)\|_{2} \leq\|F(T)\|_{1}=\sup _{Y \in \mathbb{R}^{n}}\|\boldsymbol{f}(t, Y)\|_{1} \leq$ $\|\omega(T)\|=2 T^{2}=0.72,\|\Phi\|_{2}=0.4473, C_{\Phi}=\frac{\|F(T)\|_{1}}{\|\Phi\|_{2}}=1.61, C_{0}=\frac{1}{\Gamma(0.6)}=1.11917, T^{0.6}=$ $0.6^{0.6}=0.736022$ and $E_{0.6}\left(0.5 * 0.6^{0.6}\right)=1.57201$. Then if $\delta=\|\Phi\|_{2}=0.4473$ we obtain that $\|X(T)\|_{2}=\|X(0.6)\|_{2}=1.64291$. Using the same $\delta=0.61$ as in [25] we obtain that $\|X(T)\|_{2}=\|X(0.6)\|_{2}=1.89127$. Then applying Theorem 9 we obtain that (55) is finite-time stable with respect to $\left\{0, J_{T}, \delta, \varepsilon, \sigma\right\}$ when $\varepsilon \geq 1.89127$.

Please note that our result is better than the best result given in Table 1 in [25] and hence our estimation (47) is more accurate than the estimations (12) and (13) used for the best results in Table 1.

Example 3. Consider

$\begin{cases}D_{0+}^{\alpha} X(t)=A_{0}(t) X(t)+A_{1}(t) X(t-\sigma(t))+D w(t)+f(t, X(t), X(t-\sigma(t), w(t))), & t>0 \\ X(t)=\Phi(t), & t \in[-\sigma, 0]\end{cases}$

The IP (56) is considered in [26] for $A_{0}=\left(\begin{array}{cc}0 & 1 \\ -2 & 0\end{array}\right), A_{1}=\left(\begin{array}{ll}0 & 0 \\ 3 & 4\end{array}\right), D=\left(\begin{array}{l}1 \\ 0\end{array}\right), w(t) \in$ $C\left(\overline{\mathbb{R}}_{+}, \mathbb{R}^{n}\right)$ with $\|w(t)\|_{2}=0.1, \alpha=0.5, T=5, \delta=0.1$ and $\sigma(t)=0.1 \sin ^{2} t$. For simplicity we will assume that $f(t, X(t), X(t-\sigma(t), w(t))) \equiv 0, t \in \overline{\mathbb{R}}_{+}$. Then via (47) we obtain that $\|X(5)\|_{2}=1.95384 E+106$ and then (55) is finite-time stable with respect to $\left\{0, J_{T}, \delta, \varepsilon, \sigma\right\}$ when $\varepsilon \geq 1.95384 E+106$, which result coincides with the result calculated by us for this case via condition (5) in [27].

\section{Conclusions}

As was mentioned above, in this work we set out some considerations illustrating our point of view concerning the different sources of the impacts of the finite-time stability. It is easy to see that they appear not only as an influence on the finite-time stability connecting with the impact of the aftereffect (the delay effect) described in the mathematical model through the initial function and the fractional derivatives, but it seems to be reasonable to include into account the impact of external influences too. From a physical point of view, we can interpret as an influence of external forces the existence in the model different kind of functions $F\left(t, X(t), X_{t}(\theta)\right)$, etc..., mathematically understood as nonlinear perturbations. Namely, if we apply the formal definition to the nonhomogeneous system (1), when $F(t) \not \equiv \mathbf{0}$ for $t \in J_{T}$ and $\|\Phi\|=0$ we obtain a case when the inequality $\|\Phi\|<\delta$ is fulfilled for all $\delta \in \mathbb{R}_{+}$but this fact is not useful to establish the possible existing finite-time stability.

Our attempt to clarify which impact is leading for the process, the impact hereditary of the process expressed by $\|\Phi\|$, the impact of the outer perturbations expressed by $\|F(T)\|$, or the complex of both factors expressed by the ratio $C_{\Phi}=\|\Phi\|^{-1}\|F(T)\|$ imposes a more detailed study not only of the homogeneous case when $\|F(T)\|=0$, but also the important case when $\|\Phi\|=0$. This reason focuses our attention on the case of the nonhomogeneous system with $\|\Phi\|=0$ and it was very strange for us that we could not find some extra consideration of this case. Please note that conditions of the type "there exists $M \in \mathbb{R}_{+}$, such that $\|\Phi\|^{-1}\|F(T)\| \leq M$ " are often used without to clime that $\|\Phi\| \neq 0$.

The result from this study is in general a pure mathematical answer, that is the mean by the construction of the proofs, we must limit the impact of $\|\Phi\|$ and $\|F(T)\|$ to linear or no more than power-law growth as in the right side of the estimation (23) and avoid the high nonlinear impact of $C_{\Phi}=\|\Phi\|^{-1}\|F(T)\|$ if it is involved as an argument in the Mittag-Leffler function as in estimation (20). 
Our comparison between the two most used approaches leads to the following conclusions: The most accurate estimation can be obtained by direct numerical calculation from the integral representation of the solutions, but before them, it is needed to simplify symbolically these presentations, which essentially increase the accuracy of the results (see Example 54).

Since the estimation via Mittag-Leffler functions of the fundamental matrices involved in the integral representation are not accurate enough, then generally speaking we cannot unequivocally point to one of the compared methods as better. It seems from the examples that this maybe, in general, be not possible, because it depends essentially also from the possibility to have explicit presentation of the fundamental matrices.

Author Contributions: Conceptualization, H.K., M.V., E.M. and A.Z. Writing-Review and Editing, H.K., M.V., E.M. and A.Z. All authors contributions in the article are equal. All authors have read and agreed to the published version of the manuscript.

Funding: This research was partially supported by project FP21-FMI-002 of the Scientific Fund of the University of Plovdiv Paisii Hilendarski, Bulgaria. The third AUTHOR (E.M.) is supported by the Bulgarian Ministry of Education and Science under the National Research Program "Young scientists and postdoctoral students", Stage III-2021/2022.

Institutional Review Board Statement: Not applicable.

Informed Consent Statement: Not applicable.

Data Availability Statement: Not applicable.

Conflicts of Interest: The authors declare no conflict of interest.

\section{References}

1. Kilbas, A.A.; Srivastava, H.M.; Trujillo, J.J. Theory and Applications of Fractional Differential Equations; Elsevier Science B.V: Amsterdam, The Netherlands, 2006.

2. Podlubny, I. Fractional Differential Equation; Academic Press: San Diego, CA, USA, 1999.

3. Jiao, Z.; Chen, Y.Q.; Podlubny, I. Distributed-Order Dynamic Systems: Stability, Simulation, Applications and Perspectives; Springer: Berlin, Germany, 2012.

4. Diethelm, K. The Analysis of Fractional Differential Equations, an Application-Oriented Exposition Using Differential Operators of Caputo Type; Lecture Notes in Mathematics; Springer: Berlin, Germany, 2010; Volume 2004.

5. Stamova, I.; Stamov, G. Functional and Impulsive Differential Equations of Fractional Order; Qualitative Analysis and Applications; CRC Press: Boca Raton, FL, USA, 2017.

6. Jiang, S.; Zhang, J.; Zhang, Q.; Zhang, Z. Fast evaluation of the Caputo fractional derivative and its applications to fractional diffusion equations. Commun. Comput. Phys. 2017, 21, 650-678. [CrossRef]

7. $\mathrm{Gu}, \mathrm{X} .-\mathrm{M} . ; \mathrm{Wu}, \mathrm{S}$.-L. A parallel-in-time iterative algorithm for Volterra partial integro-differential problems with weakly singular kernel. J. Comput. Phys. 2020, 471, 109576. [CrossRef]

8. Li, C.P.; Zhang, F.R. A survey on the stability of fractional differential equations. Eur. Phys. J. Spec. Top. 2011, 193, 27-47. [CrossRef]

9. Krol, K. Asymptotic properties of fractional delay differential equations. Appl. Math. Comput. 2011, 218, 1515-1532. [CrossRef]

10. Zhang, F.R.; Li, C.P. Stability Analysis of Fractional Differential Systems with Order Lying in (1, 2). Adv. Differ. Equ. 2011, 1-17. [CrossRef]

11. Veselinova, M.; Kiskinov, H.; Zahariev, A. Stability analysis of linear fractional differential system with distributed delays. AIP Conf. Proc. 2015, 1690, 040013-1-040013-8.

12. Veselinova, M.; Kiskinov, H.; Zahariev, A. Explicit conditions for stability of neutral linear fractional system with distributed delays. AIP Conf. Proc. 2016, 1789, 040005-1-040005-13.

13. Cermak, J.; Hornicek, J.; Kisela, T. Stability regions for fractional differential systems with a time delay. Commun. Nonlinear Sci. Numer. Simul. 2015, 31, 108-123. [CrossRef]

14. Cong, N.D.; Doan, T.S.; Siegmund, S.; Tuan, H.T. Linearized asymptotic stability for fractional differential equations. Electron. J. Qual. Theory Diff. Equ. 2016, 39, 1-13. [CrossRef]

15. Boyadzhiev, D.; Kiskinov, H.; Veselinova, M.; Zahariev, A. Stability analysis of linear distributed order fractional systems with distributed delays. Fract. Calc. Appl. Anal. 2017, 20, 914-935. [CrossRef]

16. Liu, S.; Zhou, X.; Li, X.; Jiang, W. Asymptotical stability of Riemann-Liouville fractional singular systems with multiple time-varying delays. Appl. Math. Lett. 2017, 65, 32-39. [CrossRef] 
17. Zahariev, A.; Kiskinov, H. Asymptotic stability of the solutions of neutral linear fractional system with nonlinear perturbation. Mathematics 2020, 8, 390. [CrossRef]

18. Kaslik, E.; Sivasundaram, S. An analytical and numerical methods for the stability analysis of linear fractional delay differential equations. J. Comput. Appl. Math. 2012, 236, 4027-4041. [CrossRef]

19. Kamenkov, G. On stability of motion over a finite interval of time. J. Appl. Math. Mech. (PMM) 1953, 17, 529-540. (In Russian)

20. Dorato, P. An Overview of Finite-Time Stability; Current Trends in Nonlinear Systems and Control; Birkhaeuser: Boston, MA, USA, 2006; pp. 185-194.

21. Lazarevic, M.P.; Spasic, A.M. Finite-time stability analysis of fractional order time-delay systems: Gronwall's approach. Math Comput. Model. 2009, 49, 475-481. [CrossRef]

22. Zhang, X. Some results of linear fractional order time-delay system. Appl. Math. Comput. 2008, 197, 407-411. [CrossRef]

23. Ma, Y.; Wu, B.; Wang, Y. Finite-time stability and finite-time boundedness of fractional order linear systems. Neurocomputing 2016, 173, 2076-2082. [CrossRef]

24. Li, M.; Wang, J. Finite time stability of fractional delay differential equations. Appl. Math. Lett. 2017, 64, 170-176. [CrossRef]

25. Li, M.; Wang, J. Exploring delayed Mittag-Leffler type matrix functions to study finite time stability of fractional delay differential equations. Appl. Math. Comput. 2018, 324, 254-265. [CrossRef]

26. Phat, N.; Thanhb, N. New criteria for finite-time stability of nonlinear fractional-order delay systems: A Gronwall inequality approach. Appl. Math. Lett. 2018, 83, 169-175. [CrossRef]

27. Zhang, F.; Qian, D.; Li, C. Finite-time stability analysis of fractional differential systems with variable coefficients. Chaos 2019, 29, 013110. [CrossRef] [PubMed]

28. Du, F.; Jia, B. Finite-time stability of a class of nonlinear fractional delay difference systems. Appl. Math. Lett. 2019, 98, 233-239. [CrossRef]

29. Du, F.; Lu, J.G. New criterion for finite-time stability of fractional delay systems. Appl. Math. Lett. 2020, 104, 106248. [CrossRef]

30. Du, F.; Jia, B. Finite-time stability of nonlinear fractional order systems with a constant delay. J. Nonlinear Model. Anal. 2020, 2,1-13.

31. Kiskinov, H.; Madamlieva, E.; Veselinova, M.; Zahariev, A. Existence of Absolutely Continuous Fundamental Matrix of Linear Fractional System with Distributed Delays. Mathematics 2021, 9, 150. [CrossRef]

32. Horn, R.A.; Johnson, C.R. Matrix Analysis, 2nd ed.; Cambridge University Press: Cambridge, UK, 2013.

33. Boyadzhiev, D.; Kiskinov, H.; Zahariev, A. Integral representation of solutions of fractional system with distributed delays. Integral Transf. Special Funct. 2018, 29, 725-744. [CrossRef]

34. Zahariev, A.; Kiskinov, H.; Angelova, E. Linear fractional system of incommensurate type with distributed delay and bounded Lebesgue measurable initial conditions. Dyn. Syst. Appl. 2019, 28, 491-506.

35. Ye, H.; Gao, J.; Ding, Y. A generalized Gronwall inequality and its application to a fractional differential equation. J. Math. Anal. Appl. 2007, 328, 1075-1081. [CrossRef] 